Research Paper

\title{
Preoperative LMR and Serum CA125 Level as Risk Factors for Advanced Stage of Ovarian Cancer
}

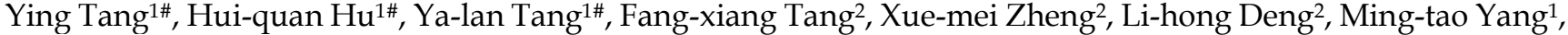 \\ Su Yin ${ }^{1}$, Jun $\mathrm{Li}^{1}$, Fan $\mathrm{Xu}^{1 凶}$ \\ 1. Department of Obstetrics and Gynecology, The Affiliated Nanchong Central Hospital of North Sichuan Medical College, Nanchong, Sichuan, China \\ 2. North Sichuan Medical College, Nanchong, Sichuan, China. \\ \#These authors contributed equally to this work. \\ $\bowtie$ Corresponding author: Fan Xu, E-mail: xufanyidingxin@163.com. Phone: +86-15984877283. \\ (c) The author(s). This is an open access article distributed under the terms of the Creative Commons Attribution License (https://creativecommons.org/licenses/by/4.0/). \\ See http://ivyspring.com/terms for full terms and conditions.
}

Received: 2021.04.27; Accepted: 2021.07.29; Published: 2021.08.09

\begin{abstract}
Objectives: This study was to analyze the relationships between lymphocyte-to-monocyte ratio (LMR) alone or combined with serum CA125 (COLC) and advanced stage of ovarian cancer (OC).

Methods: The receiver-operating characteristic (ROC) curves of LMR, CA125, and COLC staging OC were constructed by a retrospective study. Furthermore, a binary logistic regression model was used to assay the independent risk factors for OC staging.

Results: Two hundred and twenty-five patients with $O C$ were identified in this cohort. Eighty-five OC patients were diagnosed at an early stage, and 140 OC patients were diagnosed at an advanced stage. The median of LMR in the early stage was higher than that in advanced stage (4.4 vs. 2.8), and the median of serum CA1 25 was lower than that in advanced stage $(80 \mathrm{U} / \mathrm{mL}$ vs. $251.3 \mathrm{U} / \mathrm{mL})$. Multivariate logistic regression $\mathrm{LMR} \leq 3.7$ $(\mathrm{OR}=0.299,95 \% \mathrm{Cl}: 0.093-0.962, \mathrm{P}=0.043)$ and $\mathrm{CA} 125>95.7 \mathrm{U} / \mathrm{mL}(\mathrm{OR}=4.317,95 \% \mathrm{Cl}: 1.436-12.977$, $\mathrm{P}=0.009$ ) were risk factors for stage of advanced $\mathrm{OC}$ whether presence or absence of malignant ascites. Furthermore, the area under the curve of COLC was higher than that of LMR $(0.782$ vs. 0.732$)$ or serum CA1 25 (0.782 vs. 0.708$)$ in staging OC. The specificity of COLC was higher than that of LMR $(87.1 \%$ vs. $70.6 \%)$ or serum CA125 (87.1\% vs. $61.2 \%)$ in staging OC.

Conclusion: LMR alone or in combination with serum CA125 might be associated with OC staging. Besides, as a predictive factor, COLC may have a high specificity in staging OC.
\end{abstract}

Key words: Ovarian cancer, lymphocyte-to-monocyte ratio (LMR), CA125, International Federation of Gynecology and Obstetrics (FIGO), stage, combine

\section{Introduction}

Ovarian cancer $(\mathrm{OC})$ is the third most common gynecological malignancy with high mortality [1]. Owing to the tissue and anatomical characteristics [2], most OC patients are diagnosed at advanced stage (FIGO III-IV, version 2014) [3]. The stage of disease is the main prognostic factor for OC [4], paving the way for individualization of therapy, including primary debulking surgery (PDS) and neoadjuvant chemotherapy (NACT) followed by interval debulking surgery (IDS) [5]. Thus, it is an urgent need for stratifying the preoperative stage of OC. At present, $\mathrm{CT}$ and MRI are the recommended imaging modality for staging OC [6], however, they are relatively expensive, besides, the sensitivity and specificity have not reach consensus. Effective and inexpensive indexes, which can accurately reflect the preoperative stage of advanced ovarian cancer (AOC) have not reach consensus.

The lymphocyte-to-monocyte ratio (LMR), an inflammator factor, can represent the balance between lymphocyte levels and monocyte levels in OC. The LMR has recently been used to evaluate survival value in various solid cancers [7-9]. However, few studies have evaluated the magnitude of LMR on OC staging. Serum cancer antigen 125 (CA125) is a mucin-type glycoprotein, produced by the MUC16 gene, associated with the cellular membrane [10]. Previous studies have shown that serum CA125 was 
associated with the prognosis of AOC [11]. However, the relationship between serum CA125 and OC staging is still not completely understood and shows inconsistent findings.

Therefore, we assessed the magnitude of LMR alone and in combination with serum CA125 (COLC) to attempt to identify preoperative factors to stage OC.

\section{Materials and methods}

\section{Patients}

In this retrospective study, data of 225 OC patients whose initial treatment was PDS in the Affiliated Nanchong Central Hospital of North Sichuan Medical College from January 2008 to January 2021 were collected.

The inclusion criteria were as follows: (1) Chest and whole abdomen enhanced CT examination were performed before operation; (2) the suidan score[12] was less than or equal to 2; (3) the chief physician were responsible for PDS, and patients were given 6 cycles of postoperative adjuvant chemotherapy (POAC); (4) intraoperative lymphadenectomy was performed in PDS; (5) pathological diagnosis was used as the gold standard for assessing the stage of OC, and the pathology reports were obtained after evaluation and issued by two senior pathologists; (6) the complete blood count and serum CA125 values were collected before any anti-tumor therapy; (7) sufficient data could be extracted for the fourfold Table.

The exclusion criteria were as follows: (1) patients who had received anti-tumor therapy such as radiotherapy and chemotherapy before the operation; (2) the suidan score was more than 2; (3) patients with complications due to other malignant tumors except OC.

The standard procedures for PDS consisted of total hysterectomy, two attachments, omentectomy, pelvic and para-aortic lymphadenectomy sampling, appendectomy (mucinous appendicitis), routine abdominal washing and visible tumor lumps if applicable [13]. POAC, including paclitaxel (175 $\mathrm{mg} / \mathrm{m}^{2}$ ) and carboplatin (AUC 5), was initiated within 6 weeks of PDS, repeated every three weeks for 6 cycles when required [14].

All procedures performed in this study involving human participants were in accordance with the 1964 Helsinki Declaration ethical standards and approved by the Institutional Ethical Committee of the Affiliated Nanchong Central Hospital of North Sichuan Medical College. Written informed consent could not be obtained from each patient duing to the restrospective study, however, a notice about study design and contact information was posted at a public location in our hospital.

\section{Samples and marker assays}

Preoperative blood samples collected in appropriate collection tubes were transported to clinical laboratory center in hospital at room temperature to test whole blood cell count and serum CA125 level. Samples were retrieved and serum serum CA125 concentrations were measured by twostep immunoassay for the quantitative determination with flexible assay protocols [15]. All biomarkers were assayed in a central laboratory within 3 days before surgery, and the lab personnel were blinded to the clinical data.

\section{Data analysis}

Clinical variables such as age, BMI, FIGO stage, histological type and grade, malignant ascites, lymph node metastases, whole blood cell count, serum CA125 levels within 3 days before surgery were retrospectively analyzed. The LMR was calculated based on the lymphocyte count dividing monocyte count [8]. If the patient had several preoperative results of blood parameters, the most recent results before PDS were selected for analysis.

Descriptive statistics were used to show the baseline characteristics of participants in this study. Chi square test and binary logistic regression were used to analyze the relationship between clinicopathological features and OC staging. Areas under the receiver operating characteristic curves (ROCs; MedCalc Software bvba [ver. 15.2.1], Ostend, Belgium) were used to assess LMR, serum CA125, and COLC. The optimal cut-off values were determined according to ROC. BMI was categorized as $>24$ or $\leq 24$ [16]. Age was classified as $\geq 50$ or $<50$ (years) [11]; white blood cell as $>6.4$ or $\leq 6.4$ [8]. COLC was based on the Glasgow prognostic score (GPS) (Table 5). Statistical analyses were performed using SPSS software (ver. 20.0; IBM Corp, Armonk, NY, USA). A two-sided $P$ value of $<0.05$ was considered to indicate statistical significance.

\section{Results}

\section{Entire cohort characteristics}

Two hundred and twenty-five OC patients met the inclusion criteria. Eighty-five patients with OC were diagnosed at an early stage (FIGO I-II), and 140 OC patients were diagnosed at an advanced stage. The majority histologic subtype and grade of patients with OC were serous $(n=156,69.3 \%)$ and G3 $(n=135$, $60.0 \%$ ), respectively. The median (range) of LMR and serum CA125 were 3.5 (0.5-18.00) and 161 (1.8-5672.6) $\mathrm{U} / \mathrm{mL}$. The baseline characteristics of all patients with 
OC are shown in Table 1.

Table 1. Clinical characteristics of patients with ovarian cancer

\begin{tabular}{ll}
\hline Variable & Median (range) \\
\hline Age & $50(18-89)$ \\
BMI $\left(\mathrm{kg} / \mathrm{m}^{2}\right)$ & $22.9(16.5-29.8)$ \\
Histologic subtype, $\mathbf{n}(\%)$ & \\
Serous & $156(69.3)$ \\
Endometrioid & $25(11.1)$ \\
Transitional cell & $20(9.0)$ \\
Clear cell & $12(5.3)$ \\
Mucinous & $11(4.9)$ \\
Other & $1(0.4)$ \\
FIGO Stage, $\mathbf{n}(\%)$ & \\
I & $62(27.6)$ \\
II & $39(17.3)$ \\
III & $109(48.4)$ \\
IV & $15(6.7)$ \\
Histological grade, $\mathbf{n}(\%)$ & \\
G1 & $42(18.7)$ \\
G2 & $48(21.3)$ \\
G3 & $135(60.0)$ \\
Malignant ascites, $\mathbf{n}(\%)$ & \\
Yes & $134(59.6)$ \\
No & $91(40.4)$ \\
Lymph node metastases, $\mathbf{n}(\%)$ & \\
Yes & $86(38.2)$ \\
No & $139(61.8)$ \\
White blood cell ${ }^{* 10} 9$ & $7.25(3.3-16.5)$ \\
Lymphocyte*10 & $1.27(0.3-2.8)$ \\
Monocyte*10 & $0.39(0.1-1.6)$ \\
LMR & $3.5(0.5-18.0)$ \\
Serum CA125 (U/mL) & $161(1.8-5672.6)$ \\
\hline BMI, body mass index; FIGO, Federation of Gynecologists and Obstetricians; LMR, \\
lymphocyte/monocyte ratio; CA125, cancer antigen 125.
\end{tabular}

\section{Analysis of ROC curves of the LMR and CA125}

The ROC curve of LMR staging OC is shown in Figure 1a. The optimal cut-off value of LMR was 3.7, and the sensitivity and specificity were $70.7 \%$ and $70.6 \%$, respectively. Patients with OC were divided into LMR $>3.7$ group $(\mathrm{n}=101,44.9 \%)$ and $\mathrm{LMR} \leq 3.7$ group $(\mathrm{n}=124,55.1 \%)$.

The ROC curve of serum CA125 staging OC is shown in Figure $1 \mathrm{~b}$. The optimal cut-off value of serum CA125 was $95.7 \mathrm{U} / \mathrm{mL}$. The sensitivity and specificity were $80.0 \%$ and $61.2 \%$, respectively. OC patients were divided into CA125 $>95.7 \mathrm{U} / \mathrm{mL}$ group $(\mathrm{n}=145,64.4 \%)$ and $\mathrm{CA} 125 \leq 95.7 \mathrm{U} / \mathrm{mL}$ group $(\mathrm{n}=80$, $35.6 \%)$.

\section{Clinical characteristics of OC between early and advanced stage}

Clinicopathological features between early and advanced stage of OC were compared by chi-square test. The median of LMR in early stage was higher than that in advanced stage (4.4 vs. 2.8), and the median of serum CA125 was lower in the early stage than that in advanced stage $(80 \mathrm{U} / \mathrm{mL}$ vs. 251.3 $\mathrm{U} / \mathrm{mL}$ ). These results showed that $\mathrm{BMI} \geq 24$, histologic serous and G1, malignant ascites (+), lymph node metastases $(+), \mathrm{LMR} \leq 3.7, \mathrm{CA} 125>95.7 \mathrm{U} / \mathrm{mL}$ were all associated with AOC $(\mathrm{P}<0.05)$ (Table 2$)$.

Table 2. Clinical characteristics of ovarian cancer between early stage and advanced stage

\begin{tabular}{|c|c|c|c|c|c|c|}
\hline \multirow[t]{2}{*}{ Variable } & \multicolumn{2}{|c|}{ Early stage $(n=85)$} & \multicolumn{2}{|c|}{ Advanced stage $(n=140)$} & \multirow[t]{2}{*}{$\mathrm{X} 2$} & \multirow{2}{*}{$\begin{array}{l}\mathrm{P} \\
\text { value }\end{array}$} \\
\hline & $\mathrm{n}(\%)$ & Middle (Rang) & $\mathrm{n}(\%)$ & Middle (Rang) & & \\
\hline \multicolumn{5}{|c|}{ Age (years) } & 0.414 & 0.520 \\
\hline$<50$ & $43(50.6)$ & 43 (18 49) & $77(55.0)$ & $43(23 \sim 49)$ & & \\
\hline$\geq 50$ & $42(49.4)$ & $58(50 \sim 89)$ & $63(45.0)$ & $60(50 \sim 80)$ & & \\
\hline \multicolumn{5}{|c|}{ BMI $\left(\mathbf{k g} / \mathbf{m}^{2}\right)$} & 4.703 & 0.03 \\
\hline$<24$ & $39(45.9)$ & $20.96(17.1 \sim 23.8)$ & $85(60.7)$ & $25.6(24.0 \sim 29.8)$ & & \\
\hline$\geq 24$ & $46(65.1)$ & $24.79(24.0 \sim 29.8)$ & $55(39.3)$ & $20.9(16.5 \sim 23.8)$ & & \\
\hline \multicolumn{7}{|c|}{ Histologic subtype, $\mathbf{n}(\%)$} \\
\hline Serous & $43(50.6)$ & & $\begin{array}{l}108 \\
(77.1)\end{array}$ & & 16.897 & $<0.001$ \\
\hline $\begin{array}{l}\text { Non- } \\
\text { serous }\end{array}$ & $42(49.4)$ & & $34(22.9)$ & & & \\
\hline \multicolumn{7}{|c|}{ Histological grade, $\mathbf{n}(\%)$} \\
\hline G1 & $59(69.4)$ & & $\begin{array}{l}123 \\
(54.7)\end{array}$ & & 16.897 & 0.001 \\
\hline G2/G3 & $26(30.6)$ & & $17(45.3)$ & & & \\
\hline \multicolumn{5}{|c|}{ Malignant ascites, $\mathbf{n}(\%)$} & 51.533 & $<0.001$ \\
\hline Yes & \multicolumn{2}{|l|}{$25(29.4)$} & $\begin{array}{l}109 \\
(77.9)\end{array}$ & & & \\
\hline No & $60(70.6)$ & & $31(22.1)$ & & & \\
\hline \multicolumn{5}{|c|}{ Lymph node metastases, $\mathrm{n}(\%)$} & 40.497 & $<0.001$ \\
\hline Yes & $10(11.8)$ & & $76(33.8)$ & & & \\
\hline No & $75(88.2)$ & & $31(66.2)$ & & & \\
\hline \multicolumn{5}{|c|}{ White blood cell ${ }^{*} 10^{9}$} & 0.213 & 0.644 \\
\hline$<6.40$ & 28 & $5.5(3.6 \sim 6.4)$ & $42(60.7)$ & $5.35(3.3 \sim 6.4)$ & & \\
\hline$\geq 6.40$ & 57 & $7.7(6.4 \sim 16.5)$ & $98(39.3)$ & $8.63(6.4 \sim 16.2)$ & & \\
\hline LMR & & & & & 36.470 & $<0.001$ \\
\hline$\leq 3.7$ & $25(11.8)$ & $2.43(0.9 \sim 3.6)$ & $99(33.8)$ & $2.15(0.5 \sim 3.7)$ & & \\
\hline$>3.7$ & $60(88.2)$ & $4.93(3.7 \sim 18.0)$ & $41(66.2)$ & $4.93(3.7 \sim 11.6)$ & & \\
\hline \multicolumn{5}{|c|}{ Serum CA125 (U/mL) } & 39.13 & $<0.001$ \\
\hline$\leq 95.7$ & 52 & 34.65 (2.2 95.7) & 28 & $22.4(1.8 \sim 74.7)$ & & \\
\hline$>95.7$ & 33 & 342.5 (98 1401) & 112 & $\begin{array}{l}423.5 \\
(95.8 \sim 5672.6)\end{array}$ & & \\
\hline
\end{tabular}

BMI, body mass index; LMR, lymphocyte/monocyte ratio; CA125, cancer antigen 125.

\section{Factors influencing stage}

Association of clinicopathological factors of OC patients and OC staging were analyzed by univariate and multivariate binary logistic regression analyses. In univariate analyses, histological subtype and grade, malignant ascites, lymph node metastases $(+)$, $\mathrm{LMR} \leq 3.7$, and CA125>95.7 $\mathrm{U} / \mathrm{mL}$ were all associated with advanced stage in patients with $\mathrm{OC}(\mathrm{P}<0.05)$ (Table 3). In the multivariate logistic regression, malignant ascites (OR=3.917, 95\% CI 1.560-9.833, $\mathrm{P}=0.004)$; lymph node metastases (OR=5.338, 95\% CI 2.356-12.093, $\mathrm{P}=0.004)$; $\mathrm{LMR} \quad(\mathrm{OR}=0.314,95 \% \mathrm{CI}$ $0.143-0.687, \mathrm{P}=0.004)$; and serum CA125 (OR=4.045, 95\% CI 1.883-8.692, $\mathrm{P}<0.001)$ were associated with AOC (Table 3). Multivariate logistic regression for presence of malignant ascites $(n=134)$ or absence of malignant ascites $(\mathrm{n}=91)$ among matched samples is shown in Table 4. The results showed that LMR $\leq 3.7(\mathrm{OR}=0.299$, 95\% CI 0.093-0.962, $\mathrm{P}=0.043)$ and CA125>95.7 U/mL $(\mathrm{OR}=4.317,95 \%$ CI 1.436-12.977, $\mathrm{P}=0.009)$ were risk factors for stage of AOC (Table 4). 
a

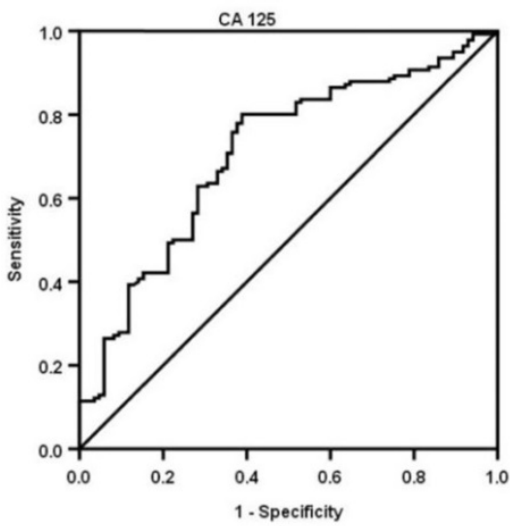

b

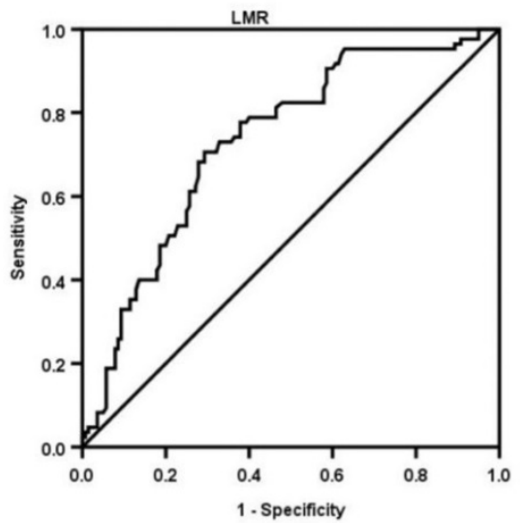

c

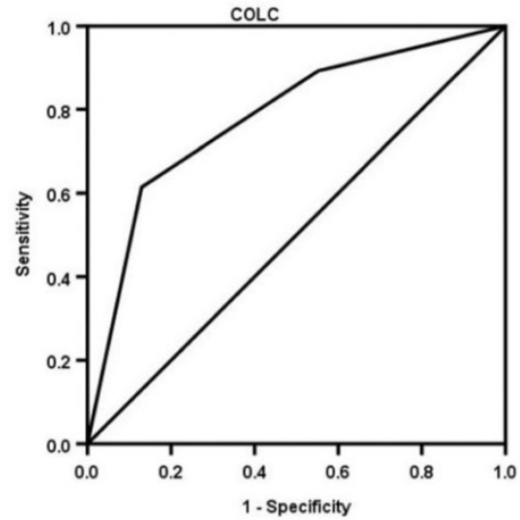

Figure 1. Receiver-operating characteristic curve analyses of LMR (a), CA125 (b), and COLC (c) in OC patients. The AUC of COLC was higher than that of LMR (0.782 vs. 0.732 ) or CA125 (0.782 vs.0.708), the specificity of COLC was higher than that of LMR (87.1\% vs. $70.6 \%)$ or CA 125 (87.1\% vs. $61.2 \%)$. AUC, area under the curve; LMR, lymphocyte/monocyte ratio; CA125, cancer antigen 125 ; COLC, combination of LMR and CA125.

Table 3. Binary logistic regression analysis of ovarian cancer staging

\begin{tabular}{|c|c|c|c|c|c|c|}
\hline \multirow[t]{2}{*}{ Variables } & \multicolumn{3}{|c|}{ Univariate } & \multicolumn{3}{|c|}{ Multivariate } \\
\hline & OR & $95 \% \mathrm{CI}$ & $\mathrm{P}$ & OR & $95 \% \mathrm{CI}$ & $\mathrm{P}$ \\
\hline $\begin{array}{l}\text { Age (age) } \\
\text { (>50 vs. } \leq 50)\end{array}$ & 1.014 & $(0.591-1.738)$ & 0.961 & 0.730 & $(0.339 \sim 1.571)$ & 0.422 \\
\hline $\begin{array}{l}\text { BMI }\left(\mathrm{kg} / \mathrm{m}^{2}\right) \\
(\geq 24 \text { vs. }<24)\end{array}$ & 1.002 & $(0.421-2.386)$ & 0.996 & 0.803 & $(0.377 \sim 1.710)$ & 0.569 \\
\hline Histological subtype (serous vs. others) & 3.188 & $(1.606-6.329)$ & 0.001 & 2.120 & $(0.980 \sim 4.585)$ & 0.056 \\
\hline $\begin{array}{l}\text { Histological grade } \\
\text { (G2-G3 vs. G1) }\end{array}$ & 0.829 & $(0.482-1.423)$ & 0.496 & 1.275 & $(0.488 \sim 3.335)$ & 0.620 \\
\hline Malignant ascites (yes vs no) & 8.439 & $(4.567-15.591)$ & $<0.001$ & 3.917 & $(1.560 \sim 9.833)$ & 0.004 \\
\hline $\begin{array}{l}\text { Lymph node metastases } \\
\text { (yes vs no) }\end{array}$ & 8.906 & $(4.254-18.646)$ & $<0.001$ & 5.338 & $(2.356 \sim 12.093)$ & $<0.001$ \\
\hline White blood cell $\left({ }^{*} 10^{9}\right)(>6.4$ vs. $\leq 6.4)$ & 0.755 & $(0.314-1.817)$ & 0.530 & 0.597 & $(0.262 \sim 1.359)$ & 0.219 \\
\hline LMR (>3.7 vs. $\leq 3.7$ ) & 5.795 & $(3.207-10.53)$ & $<0.001$ & 0.314 & $(0.143 \sim 0.687)$ & 0.004 \\
\hline Serum CA125 (U/mL) ( $\leq 95.7$ vs.>95.7) & 6.303 & $(3.454-11.502)$ & $<0.001$ & 4.045 & $(1.883 \sim 8.692)$ & $<0.001$ \\
\hline
\end{tabular}

BMI, body mass index; LMR, lymphocyte/monocyte ratio; CA125, cancer antigen 125.

Table 4. Multivariate logistic regression analysis for presence or absence of malignant ascites of ovarian cancer among matched samples

\begin{tabular}{|c|c|c|c|c|c|c|}
\hline \multirow[t]{2}{*}{ Variables } & \multicolumn{3}{|c|}{ Malignant ascites $(+)(n=134)$} & \multicolumn{3}{|c|}{ Malignant ascites (-) $(\mathrm{n}=91)$} \\
\hline & $\overline{\mathrm{OR}}$ & $95 \% \mathrm{CI}$ & $\mathrm{P}$ & OR & $95 \% \mathrm{CI}$ & $\mathrm{P}$ \\
\hline $\begin{array}{l}\text { Age (age) } \\
(>50 \text { vs. } \leq 50)\end{array}$ & 0.512 & $(0.152 \sim 1.723)$ & 0.280 & 1.219 & $(0.412 \sim 3.609)$ & 0.721 \\
\hline $\begin{array}{l}\text { BMI }\left(\mathrm{kg} / \mathrm{m}^{2}\right) \\
(\geq 24 \text { vs. }<24)\end{array}$ & 0.986 & $(0.314 \sim 3.099)$ & 0.981 & 0.623 & $(0.213 \sim 1.827)$ & 0.389 \\
\hline $\begin{array}{l}\text { Histological subtype } \\
\text { (serous vs. others) }\end{array}$ & 2.595 & $(0.750 \sim 8.974)$ & 0.132 & 2.067 & $(0.732 \sim 5.838)$ & 0.170 \\
\hline $\begin{array}{l}\text { Histological grade } \\
\text { (G2-G3 vs. G1) }\end{array}$ & 2.755 & $(0.637 \sim 11.922)$ & 0.175 & 0.484 & $(0.127 \sim 1.850)$ & 0.289 \\
\hline $\begin{array}{l}\text { Lymph node metastases } \\
\text { (yes vs no) }\end{array}$ & 4.344 & $(1.104 \sim 17.088)$ & 0.036 & 2.916 & $(0.705 \sim 12.061)$ & 0.140 \\
\hline $\begin{array}{l}\text { White blood cell }\left({ }^{*} 10^{9}\right) \\
(>6.4 \text { vs. } \leq 6.4)\end{array}$ & 0.406 & $(0.089 \sim 1.853)$ & 0.244 & 0.682 & $(0.234 \sim 1.988)$ & 0.483 \\
\hline $\begin{array}{l}\text { LMR } \\
\text { (>3.7 vs. } \leq 3.7 \text { ) }\end{array}$ & 0.254 & $(0.076 \sim 0.849)$ & 0.026 & 0.299 & $(0.093 \sim 0.962)$ & 0.043 \\
\hline $\begin{array}{l}\text { Serum CA125 }(\mathrm{U} / \mathrm{mL}) \\
(\leq 95.7 \text { vs. }>95.7)\end{array}$ & 3.824 & $(1.163 \sim 12.570)$ & 0.027 & 4.317 & (1.436 12.977) & 0.009 \\
\hline
\end{tabular}

BMI, body mass index; LMR, lymphocyte/monocyte ratio; CA125, cancer antigen 125; +, presence of malignant ascites; -, absence of malignant ascites.

As shown above, both LMR and serum CA125 were related to OC staging. However, whether COLC had the same efficacy needed further investigation. We defined COLC per the GPS (Table 5). The ROC curve of COLC in staging OC was constructed (Figure 1c). The capacities of LMR, serum CA125 and COLC in staging OC patients were compared by ROC curves. The result showed that the AUC of COLC was 0.779 (95\% CI 0.719-0.831, $\mathrm{P}<0.001$, Figure 1c), the sensitivity and specificity of COLC were $61.4 \%$ and $87.1 \%$, respectively. The specificity of COLC was higher than that of LMR $(87.1 \%$ vs. $70.6 \%$, Figure 1a, 
1b) or CA125 $(87.1 \%$ vs. $61.2 \%$, Figure $1 b, 1 c)$ in staging OC.

Table 5. Prediction scores of LMR, CA 125 and COLC in ovarian cancer

\begin{tabular}{ll}
\hline & Score \\
\hline LMR & \\
$\leq 3.7$ & 1 \\
$>3.7$ & 0 \\
Serum CA125 $(\mathrm{U} / \mathrm{mL})$ & \\
$\leq 95.7$ & 0 \\
$>95.7$ & 1 \\
COLC & \\
LMR $>3.7$ and CA125 $<95.7 \mathrm{U} / \mathrm{mL}$ & 0 \\
LMR $>3.7$ and CA125 $>95.7 \mathrm{U} / \mathrm{mL}$ & 1 \\
LMR $\leq 3.7$ and CA125 $\leq 95.7 \mathrm{U} / \mathrm{mL}$ & 2 \\
LMR $\leq 3.7$ and CA125 $>95.7 \mathrm{U} / \mathrm{mL}$ & 3 \\
\hline LMR, lymphocyte/monocyte ratio; CA125, cancer antigen $125 ;$ COLC, combination \\
of LMR and CA125.
\end{tabular}

\section{Discussion}

In this study, we attempted to explore preoperative indicators to stage OC to help predict optimal debulking surgery, reduce surgical complications and economic burden, and better evaluate the prognosis of OC patients. Multivariate logistic regression analysis showed that LMR was significantly associated with stage of OC (Table 3): the lower the LMR, the higher the stage, and the worse the prognosis, which was consistent with the conclusion of previous research [17]. Our study also showed that serum CA125 was associated with stage of OC (Table 3); however, the specificity was lower than LMR. To better analyze the factors associated with OC staging, we recommended COLC to improve the accuracy, which achieved a higher AUC and specificity than LMR or CA125, indicating that COLC might have a higher specificity for OC staging.

Our study also showed that ascites was associated with stage of OC (Table 3), one probable mechanisms is that constitutive expression of STAT3 in malignant ascites plays a role in ovarian tumor progression and metastasis [18]. Besides, Matt et al. [19] showed that ascites might be associated with serum CA125 by regulating MUC16 expression at a posttranscriptional level through an Akt-dependent pathway. Accordingly, we matched samples with presence or absence of malignant ascites, and the results showed that both LMR and serum CA125 were significantly associated with OC staging whether presence or absence of malignant ascites (Table 4).

LMR and serum CA125 are calculated directly or computed from blood and can be easily measured, in addition to being practical and inexpensive metrics. At present, several studies have been conducted with respect to the application of LMR and serum CA125 in
OC. LMR and serum CA125 could likely serve as clinically useful indicators of metastasis and survival in OC patients $[8,11,20]$. Serum CA125 also was reported as a risk factor in the diagnosis of OC [10]. However, few studies have been conducted with respect to the magnitude of LMR serum CA125 in staging OC. Our study preliminarily showed that LMR, CA125, and COLC might be risk factors for AOC staging.

The mechanisms underlying the capacity of LMR in staging OC have not yet been elaborated. We tried to explain the possible mechanisms. First, the association between lymphocytes and malignancies has been well established. Tumor infiltrating lymphocytes in OC can prevent cancer cells from spreading and metastasis by establishing a defense barrier [21]. The decrease of peripheral blood lymphocyte count may lead to weak and insufficient tumor immune response, thereby promoting tumor progression and metastasis [22], resulting in rapid disease progression and advanced OC staging and reducing the sensitivity to chemotherapy[23, 24]. Second, inflammation can make monocytes migrate from the bone marrow into peripheral blood monocytes and differentiate into tumor-associated macrophages (TAMs) after being recruited into tumor tissue [25, 26]. TAMs can not only play an immunosuppressive role in a variety of tumor microenvironments, including $\mathrm{OC}$, but can also promote tumor cell infiltration, growth and neovascularization, and metastasis $[27,28]$. Therefore, to an extent, peripheral blood mononuclear cell counts can reflect the formation or existence of TAMs. Besides, the LMR often represents the relative decrease of lymphocyte count and (or) the relative increase of monocyte count, which can reflect the balance of anti-tumor immunity and pro-tumor inflammatory response [20]: low-level LMR often represents the dominant role of pro-tumor inflammatory response, indicating a high level of malignancy and rapid progression in OC. Furthermore, in this case, the possibility of AOC that is difficult to treat with relatively poor prognosis is higher; on the contrary, high-level LMR indicates that the anti-tumor immune system of patients with OC is more active, indicating that the disease progression of $\mathrm{OC}$ is slow, with the possibility of early stage and relatively better prognosis.

Rising and falling levels of serum serum CA125 correlate with the progression and regression of high-grade serous ovarian carcinomas [19], making serum CA125 a possible factor for OC staging. However, some researchers have also suggested that because of its low specificity and the observed increased levels in different physiological situations, 
serum CA125 is not considered as a very good differentiating biomarker for ovarian tumors [10]. Therefore, we recommended the combination of serum CA125 and LMR (COLC) to stage OC. Our results showed that the AUC and specificity of COLC were higher than those of LMR or serum CA125 to stage OC (Figure 1), implying that COLC might improve accuracy in staging $\mathrm{OC}$ and provide an advice for selection of treatment. As a new biomarker, COLC might play a role in immune surveillance and provide novel approaches and strategies for treatment of OC.

To our knowledge, this is the first study to evaluate the relationship among LMR, serum CA125, COLC and stage of OC. However, our study also has some limitations. Firstly, the retrospective design meant that we cannot conclusively state that LMR, serum CA125, and COLC are risk factors for AOC staging. However, our study provides a potential clinical strategy for staging advanced OC. Secondly, the potential bias in testing serum serum CA125 and peripheral blood level cannot be completely eliminated. Nonetheless, we believe that the influence of LMR and serum CA125 level on the risk of AOC will be of interest to clinicians to improve the accuracy in preoperative staging and make clinical strategies. Moreover, LMR is an inflammatory marker that could be affected by autoimmune status and other diseases; hence, we allowed a 30-min interval for blood collection to exclude any possible treatment or drug interference in the results.

\section{Acknowledgments}

This work was supported by the Science and Technology Department of Sichuan province Program (No. 2015JY0056), the Bureau of Science and Technology Nanchong City Program (Nos. 14A0098, 16YFZJ0029, 17YFZJ0005, 18SXHZ0126, and 18YFZJ0012).

\section{Competing Interests}

The authors have declared that no competing interest exists.

\section{References}

1. Jin $C$, Yang $M$, Han $X$, et al. Evaluation of the value of preoperative CYFRA21-1 in the diagnosis and prognosis of epithelial ovarian cancer in conjunction with CA125. Journal of ovarian research. 2019:12:114.

2. Torre L, Trabert B, DeSantis C, et al. Ovarian cancer statistics, 2018. CA Cancer J Clin. 2018;68:284-96.

3. Ataseven B, Chiva L, Harter P, et al. FIGO stage IV epithelial ovarian, fallopian tube and peritoneal cancer revisited. Gynecologic oncology. 2016;142:597-607.

4. Castellani F, Nganga E, Dumas L, et al. Imaging in the pre-operative staging of ovarian cancer. Abdominal radiology (New York). 2019;44:685-96.

5. Morgan R, Armstrong D, Alvarez R et al Ovarian Cancer, Version 12016 , NCCN Clinical Practice Guidelines in Oncology. Journal of the National Comprehensive Cancer Network : JNCCN. 2016;14:1134-63.
6. Javadi S, Ganeshan D, Qayyum A, et al. Ovarian Cancer, the Revised FIGO Staging System, and the Role of Imaging. AJR American journal of roentgenology. 2016;206:1351-60.

7. Abe S, Kawai K, Nozawa H, et al. LMR predicts outcome in patients after preoperative chemoradiotherapy for stage II-III rectal cancer. The Journal of surgical research. 2018;222:122-31.

8. Eo W, Kwon S, Koh S, et al. The Lymphocyte-Monocyte Ratio Predicts Patient Survival and Aggressiveness of Endometrial Cancer. J Cancer. 2016;7:538-45.

9. Kumarasamy C, Sabarimurugan S, Madurantakam R, et al. Prognostic significance of blood inflammatory biomarkers NLR, PLR, and LMR in cancer-A protocol for systematic review and meta-analysis. Medicine. 2019;98:e14834.

10. Dochez V, Caillon H, Vaucel E, et al. Biomarkers and algorithms for diagnosis of ovarian cancer: CA125, HE4, RMI and ROMA, a review. Journal of ovarian research. 2019;12:28

11. Yuan Q, Song J, Yang W, et al. The effect of CA125 on metastasis of ovarian cancer: old marker new function. Oncotarget. 2017;8:50015-22.

12. Suidan R, Ramirez P, Sarasohn D, et al. A multicenter assessment of the ability of preoperative computed tomography scan and CA-125 to predict gross residual disease at primary debulking for advanced epithelial ovarian cancer. Gynecologic oncology. 2017;145:27-31.

13. Gorodnova T, Sokolenko A, Kuligina E, et al. Principles of clinical management of ovarian cancer. Chinese clinical oncology. 2018;7:56.

14. Chung Y, Kim Y, Lee I, et al. Impact of neoadjuvant chemotherapy and postoperative adjuvant chemotherapy cycles on survival of patients with advanced-stage ovarian cancer. PloS one. 2017;12:e183754.

15. Harter P, Sehouli J, Lorusso D, et al. A Randomized Trial of Lymphadenectomy in Patients with Advanced Ovarian Neoplasms. The New England journal of medicine. 2019;380:822-32.

16. Yang $\mathrm{P}, \mathrm{Chou} \mathrm{C}$, Chang $\mathrm{C}$, et al. Changes in peripheral mitochondrial DNA copy number in metformin-treated women with polycystic ovary syndrome: a longitudinal study. Reproductive biology and endocrinology : RB\&E. 2020;18:69.

17. Gong J, Jiang $\mathrm{H}$, Shu $\mathrm{C}$, et al. Prognostic value of lymphocyte-to-monocyte ratio in ovarian cancer: a meta-analysis. Journal of ovarian research. 2019;12:51.

18. Saini U, Naidu S, ElNaggar A, et al. Elevated STAT3 expression in ovarian cancer ascites promotes invasion and metastasis: a potential therapeutic target. Oncogene. 2017;36:168-81.

19. Matte I, Garde-Granger P, Bessette P, et al. Ascites from ovarian cancer patients stimulates MUC16 mucin expression and secretion in human peritoneal mesothelial cells through an Akt-dependent pathway. BMC cancer. 2019;19:406

20. Tang Y, Hu H, Tang F, et al. Combined Preoperative LMR and CA125 for Prognostic Assessment of Ovarian Cancer. J Cancer. 2020;11:3165-71.

21. Li J, Wang J, Chen R, et al. The prognostic value of tumor-infiltrating $\mathrm{T}$ lymphocytes in ovarian cancer. Oncotarget. 2017;8:15621-31.

22. Gonzalez H, Hagerling C, Werb Z. Roles of the immune system in cancer: from tumor initiation to metastatic progression. Genes Dev. 2018;32:1267-84.

23. Labrosse J, Abdennebi I, Thibault L, et al. Chemosensitivity, tumor infiltrating lymphocytes (TILs), and survival of postpartum PABC patients treated by neoadjuvant chemotherapy. Breast (Edinburgh, Scotland). 2018;42:61-7.

24. Posabella A, Köhn P, Lalos A, et al. High density of CD66b in primary high-grade ovarian cancer independently predicts response to chemotherapy. Journal of cancer research and clinical oncology. 2020;146:127-36.

25. Etzerodt A, Tsalkitzi K, Maniecki M, et al. Specific targeting of CD163 TAMs mobilizes inflammatory monocytes and promotes $\mathrm{T}$ cell-mediated tumor regression. The Journal of experimental medicine. 2019;216:2394-411.

26. Szebeni G, Vizler C, Kitajka K, et al. Inflammation and Cancer: Extra- and Intracellular Determinants of Tumor-Associated Macrophages as Tumor Promoters. Mediators of inflammation. 2017;2017:9294018.

27. Zhu $\mathrm{Q}, \mathrm{Wu} X$, Wang $\mathrm{X}$. Differential distribution of tumor-associated macrophages and Treg/Th17 cells in the progression of malignant and benign epithelial ovarian tumors. Oncol Lett. 2017;13:159-66.

28. Yin $\mathrm{M}, \mathrm{Li} \mathrm{X}, \mathrm{Tan} \mathrm{S}$, et al. Tumor-associated macrophages drive spheroid formation during early transcoelomic metastasis of ovarian cancer. The Journal of clinical investigation. 2016;126:4157-73. 